\title{
Bilateral CMUT Cells and Arrays: Equivalent Circuits, Diffraction Constants, and Substrate Impedance
}

\author{
Hayrettin Köymen, Senior Member, IEEE, Abdullah Atalar, Fellow, IEEE, and A. Sinan Taşdelen
}

\begin{abstract}
We introduce the large-signal and small-signal equivalent circuit models for a capacitive micromachined ultrasonic transducer (CMUT) cell, which has radiating plates on both sides. We present the diffraction coefficient of baffled and unbaffled CMUT cells. We show that the substrate can be modeled as a very thick radiating plate on one side, which can be readily incorporated in the introduced model. In the limiting case, the reactance of this backing impedance is entirely compliant for substrate materials with a Poisson's ratio less than 1/3. We assess the dependence of the radiation performance of the front plate on the thickness of the back plate by simulating an array of bilateral CMUT cells. We find that the small-signal linear model is sufficiently accurate for large-signal excitation, for the purpose of the determining the fundamental component. To determine harmonic distortion, the large-signal model must be used with harmonic balance analysis. Rayleigh-Bloch waves are excited at the front and back surfaces similar to conventional CMUT arrays.
\end{abstract}

Index Terms-Backing impedance, bilateral, capacitive micromachined ultrasonic transducer (CMUT), diffraction constant, equivalent circuit model, hydrophone, transducer, two sided.

\section{INTRODUCTION}

$\mathbf{T}$ HE capacitive micromachined ultrasonic transducer (CMUT) technology is more than 20 years old [1]. There had been impressive progress in the theory of capacitive transducers, in the modeling approaches, both numerical and analytical, and particularly in the production technologies during these two decades. The CMUT technology is comparatively assessed with respect to other conventional technologies during this period. Despite the fact that it is a mature technology, there still remain quite few issues to be addressed, either because they are specific to CMUTs or because the enabling nature and superior performance potential of CMUT technology only recently brought these issues forward, which were unnoticed with conventional transduction approaches. Some of such issues, namely, the bilateral CMUT cell, an analytical equivalent circuit for this cell, an analytical model for

Manuscript received April 7, 2016; accepted November 10, 2016. Date of publication November 15, 2016; date of current version February 1, 2017. This work was supported by the Scientific and Technological Research Council of Turkey under Grant 114E588. The work of A. Atalar was supported by the Turkish Academy of Sciences.

H. Köymen and A. Atalar are with the Department of Electrical and Electronics Engineering, Bilkent University, 06800 Ankara, Turkey (e-mail: koymen@ee.bilkent.edu.tr).

A. S. Taşdelen is with the Bilkent University Acoustics and Underwater Technologies Research Center, Bilkent University, 06800 Ankara, Turkey.

Digital Object Identifier 10.1109/TUFFC.2016.2628882

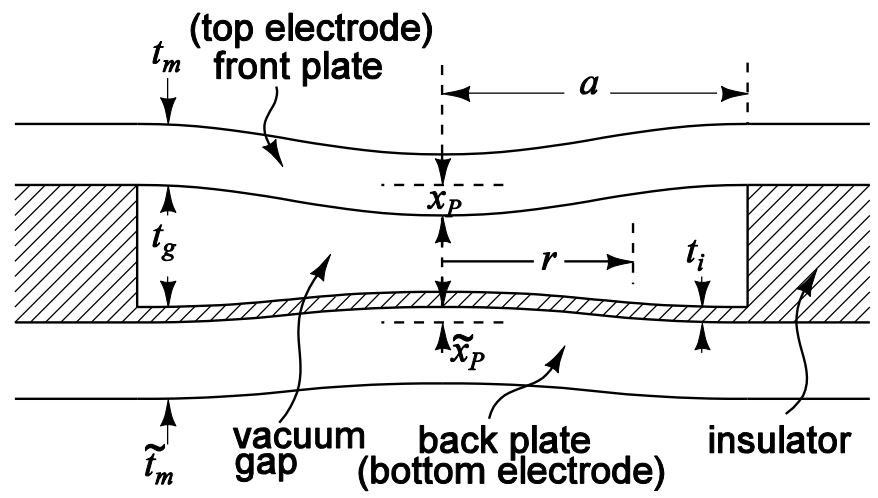

Fig. 1. Cross-sectional view of a bilateral circular CMUT cell. The radiation plates are used as the electrodes as shown, assuming conducting plate material like doped silicon.

the acoustic termination of the gap in conventional CMUT cell, and the diffraction constant of conventional and bilateral CMUT, are presented in this paper.

The applications of CMUT, hence the equivalent circuit models, are confined to cells or arrays radiating into the halfspace, where the substrate is assumed to be rigid. The extent to which the substrate can be considered rigid depends on the various parameters like the stiffness and the thickness of the substrate material, the backing material that follows the substrate, as well as the relative size of the CMUT cell or the array. Since the substrate is of finite thickness and is made of materials with finite stiffness and density, a CMUT cell can be considered as a vacuum gap with two vibrating plates on both sides, as depicted in Fig. 1. Such a bilateral CMUT can transmit into and receive from both half-spaces. In this paper, we present three-port equivalent circuit model for bilateral CMUT cells, which is an extension of the model given in [2].

We used this accurate model of the bilateral CMUT cell to derive the acoustic impedance at the back side of the gap of a conventional CMUT cell. The acoustic termination at the back side of the gap in a conventional CMUT cell has significant effects, particularly on the received signal. This effect often manifests itself as a ringing [3], [4]. The effects of substrate and other backing material when the size of the cell or an array of cells is large compared with the wavelength had been studied extensively [5]. The presented model is instrumental in analysis and quantification of the effect of the substrate material and its thickness on the backing impedance in CMUT cells. In Section III, we study the interaction of the bottom 
surface of the gap with the substrate and propose an analytical lumped model for the backing impedance presented by the substrate to the conventional CMUT cell when the size of the cell is small compared with the wavelength.

A CMUT cell with two moving plates, similar to this structure but radiating to one hemisphere, is proposed recently [6]-[8]. This new structure is referred to as "multiple moving membrane" ( $\left.\mathrm{M}^{3} \mathrm{CMUT}\right)$ and claimed to have larger swing in the upper plate at a lower dc voltage bias compared with a similar sized conventional CMUT, thus promising better performance for airborne applications. This new structure is fabricated employing a MEMSCAP sacrificial technique, PolyMUMPs [9]. The production support of this wellestablished and popular multiuser foundry service is a very important advantage for such CMUT designs using two vibrating plates. It is shown in this paper that the presented model provides a means for very accurate analysis and design of $\mathrm{M}^{3}$ CMUT.

It is shown in this paper that the bilateral CMUT cell offers advantageous applications such as omnidirectional free field microphones, hydrophones, and sources. A very high sensitivity over a very wide bandwidth is obtained in immersion for a given size, when operated at resonance or off resonance, compared with other transduction mechanisms. The bilateral CMUT is comprehensively analyzed using the presented model.

The transmission and reception characteristics of any transducer require the knowledge of relevant diffraction constants. Since CMUT cells are conventionally considered in rigid baffle, the diffraction constant is always equal to two [10] for normally incident waves. The diffraction constants for baffled and unbaffled conventional and bilateral CMUT cells are presented in Section IV, both for transmission and for reception.

Bilateral CMUT cell and arrays are simulated and the effects of different thickness of radiating plates are evaluated in Section V.

\section{Bilateral CMUT Cell Model}

The cross-sectional view of a bilateral circular CMUT cell is shown in Fig. 1, where $a$ is the diameter of the CMUT, $t_{g}$ is the gap height, $t_{i}$ is the insulating layer thickness, and $t_{m}$ and $\tilde{t}_{m}$ are the front and back plate thicknesses, respectively. The immersion medium on front and back surfaces may be different. Acoustic waves are generated in the front and back media by applying an electrical signal between the conducting plates acting as electrodes of the CMUT. The reference directions of both displacements, $x_{P}$ and $\tilde{x}_{P}$, are toward the gap. It is assumed that the plates are perfectly clamped, have uniform thickness, and operated in their mechanically linear region, both material wise and geometrically, as in [2].

\section{A. Transduction Section Model and Biasing}

We assume that the front and back surfaces have uncollapsed deflection profiles given by

$$
x(r, t)=x_{P}(t)\left(1-\frac{r^{2}}{a^{2}}\right)^{2} ; \tilde{x}(r, t)=\tilde{x}_{P}(t)\left(1-\frac{r^{2}}{a^{2}}\right)^{2}
$$

where $x_{P}(t)$ and $\tilde{x}_{P}(t)$ are the center displacements of the front and back plates, and $r$ is the radial distance from the center as shown in Fig. 1. Letting $\epsilon_{0}$ show the permittivity of the free space, the capacitance $C(t)$ between the plates can be written as an integral

$$
\begin{aligned}
C(t) & =\int_{0}^{a} \frac{\epsilon_{0} 2 \pi r}{t_{g e}-x(r, t)-\tilde{x}(r, t)} d r \\
& =\int_{0}^{a} \frac{\epsilon_{0} 2 \pi r}{t_{g e}-\left(x_{P}(t)+\tilde{x}_{P}(t)\right)\left(1-\frac{r^{2}}{a^{2}}\right)^{2}} d r
\end{aligned}
$$

where $t_{\mathrm{ge}}=t_{g}+t_{i} / \epsilon_{r}$ is the effective gap height and $\epsilon_{r}$ is the relative permittivity of the insulator. Comparing this equation with [2, eq. (2)], we see that every analytical result in [2] regarding the equivalent circuit model of transduction section is valid for the bilateral cell, if we set the total normalized center displacement as

$$
u(t)=\frac{x_{P}(t)+\tilde{x}_{P}(t)}{t_{\mathrm{ge}}} .
$$

The deflected clamp capacitance becomes

$$
C(t)=C_{0} g(u(t))
$$

where $C_{0}=\epsilon_{0} \pi a^{2} / t_{\mathrm{ge}}$ is the undeflected clamp capacitance between two plates and $g(u)$ is the transduction function as given in [2, eq. (4)].

Energy and transduction force expressions are again the same as in [2]. We define the static forces due to the ambient pressure acting on the two radiating surfaces of the CMUT cell as $F_{P b}$ and $\tilde{F}_{P b}$. These two forces are equal, if the ambient pressure is the same for two faces. The compliances of the plates to be used with the peak model are $C_{P m}$ and $\tilde{C}_{P m}$, given as

$$
C_{P m}=9 \frac{\left(1-\sigma^{2}\right) a^{2}}{16 \pi Y_{0} t_{m}^{3}}
$$

and

$$
\tilde{C}_{P m}=9 \frac{\left(1-\tilde{\sigma}^{2}\right) a^{2}}{16 \pi \tilde{Y}_{0} \tilde{t}_{m}^{3}}
$$

where $\left(Y_{0}, \sigma\right)$ and $\left(\tilde{Y}_{0}, \tilde{\sigma}\right)$ are the Young's moduli and Poisson's ratio of the front and back plate materials, respectively [2]. The respective peak static displacements, $X_{P}$ and $\tilde{X}_{P}$, are obtained as

$$
\begin{aligned}
& X_{P}=C_{P m}\left(f_{P}\left(\frac{X_{P}}{t_{\mathrm{ge}}}+\frac{\tilde{X}_{P}}{t_{\mathrm{ge}}}\right)+F_{P b}\right) \\
& \tilde{X}_{P}=\tilde{C}_{P m}\left(f_{P}\left(\frac{X_{P}}{t_{\mathrm{ge}}}+\frac{\tilde{X}_{P}}{t_{\mathrm{ge}}}\right)+\tilde{F}_{P b}\right)
\end{aligned}
$$

where $f_{P}$ is the electrostatic force function between the electrodes as defined in [2, eq. (7) and Table I], suitable for spatial peak quantities. The total normalized displacement becomes

$$
\frac{X_{P}}{t_{\mathrm{ge}}}+\frac{\tilde{X}_{P}}{t_{\mathrm{ge}}}=\frac{C_{P m}+\tilde{C}_{P m}}{t_{\mathrm{ge}}} f_{P}\left(\frac{X_{P}}{t_{\mathrm{ge}}}+\frac{\tilde{X}_{P}}{t_{\mathrm{ge}}}\right)+\frac{F_{P B}}{F_{P G}}
$$



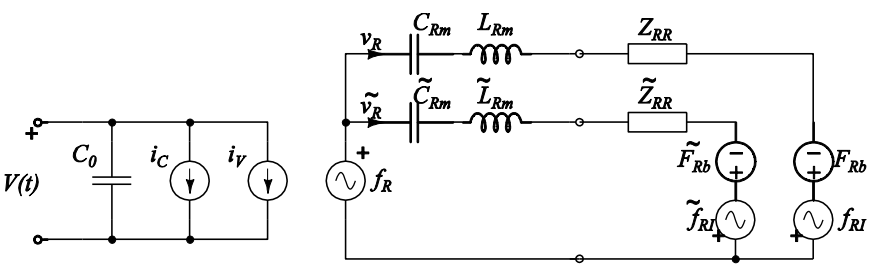

Fig. 2. Large-signal nonlinear equivalent circuit model for the bilateral CMUT cell. The velocities $v_{R}$ and $\tilde{v}_{R}$ and force $f_{R}$ in the mechanical section are spatial rms variables. $Z_{R R}$ and $\tilde{Z}_{R R}$ are the radiation impedances suitable for the rms variables.

where

$$
F_{P B}=\frac{C_{P m} F_{P b}+\tilde{C}_{P m} \tilde{F}_{P b}}{C_{P m}+\tilde{C}_{P m}}
$$

is the effective static force due to ambient pressure and

$$
F_{P G}=\frac{t_{\mathrm{ge}}}{C_{P m}+\tilde{C}_{P m}}
$$

is the force required to have the two center displacements sum up to $t_{\mathrm{ge}}$.

The collapse voltage in vacuum $V_{r}$ is given by

$$
V_{r}=\sqrt{\frac{4 t_{\mathrm{ge}}^{2}}{3 C_{0}}\left(\frac{1}{C_{P m}+\tilde{C}_{P m}}\right)} .
$$

The reference voltage, $V_{r}$, is lower compared with a one-sided CMUT cell as expected. CMUT biasing chart $(\mathrm{CBC})^{1}$ equation becomes

$$
\frac{V_{\mathrm{DC}}}{V_{r}}=\sqrt{\frac{3\left(\frac{X_{P}+\tilde{X}_{P}}{t_{\mathrm{ge}}}-\frac{F_{P B}}{F_{P G}}\right)}{2 g^{\prime}\left(\frac{X_{P}+\tilde{X}_{P}}{t_{\mathrm{ge}}}\right)}} \text { for } \frac{X_{P}+\tilde{X}_{P}}{t_{\mathrm{ge}}} \geq \frac{F_{P B}}{F_{P G}} .
$$

The variation of $V_{\mathrm{dc}}$ with respect to total normalized displacement is the same as for one-sided cell. The results obtained in [2] are directly applicable.

\section{B. Large- and Small-Signal Equivalent Circuit}

The large-signal rms equivalent circuit model [2] with the chosen directions for motion is shown in Fig. 2. The circuit parameters are exactly the same as given in [2, Table I]. ${ }^{2}$ With the same immersion media on both sides, the radiation impedances $Z_{R R}$ and $\tilde{Z}_{R R}$ are equal to each other. The compliance of rms equivalent circuit is $1 / 5$ of the compliance of the peak circuit, $C_{R m}=C_{P m} / 5$, and $\tilde{C}_{R m}=\tilde{C}_{P m} / 5$ [2]. Similarly, the inductances in the mechanical section are the masses of the front and back plates.

The small-signal equivalent circuit, shown in Fig. 3, is similar to the circuit given in [2, Fig. 6], except that the two branches for two radiating faces emerge after $-C_{\mathrm{RS}}$, the spring

\footnotetext{
${ }^{1}$ The static equilibrium for parallel plate capacitor was studied, and the equilibrium equation was plotted and discussed as equilibrium curve in [11]. The equilibrium for clamped circular plate of CMUT was derived and plotted in [2], which was later referred to as $C B C$.

${ }^{2} p_{\text {in }}$ in calculating $f_{I}$ in $\left[2\right.$, Table I] must be taken as $2 p_{\text {in }}$ instead of $p_{\text {in }}$ for waves perpendicularly incident on the CMUT surface since the model assumes that the CMUT cell is in rigid baffle [10].
}

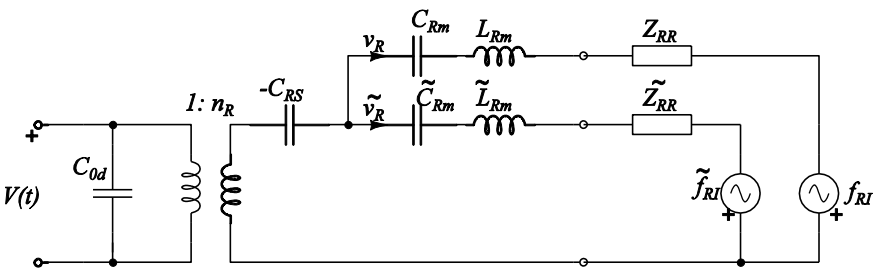

Fig. 3. Small-signal linear model of the bilateral CMUT cell. $n_{R}$ is the turns ratio of the electromechanical transformer suitable for the peak variables.

softening capacitor. The variables $n_{R}, C_{\mathrm{RS}}$, and $C_{0 d}$ are calculated as

$$
\begin{aligned}
n_{R} & =\frac{2 F_{R}}{V_{\mathrm{DC}}} \\
C_{\mathrm{RS}} & =\frac{2 t_{\mathrm{ge}}^{2}}{5 C_{00} V_{\mathrm{DC}}^{2} g^{\prime \prime}\left(X_{P} / t_{\mathrm{ge}}+\tilde{X}_{P} / t_{\mathrm{ge}}\right)} \\
& =\left(C_{R m}+\tilde{C}_{R m}\right)\left[\frac{2}{3} \frac{V_{\mathrm{DC}}^{2}}{V_{r}^{2}} g^{\prime \prime}\left(\frac{X_{P}}{t_{\mathrm{ge}}}+\frac{\tilde{X}_{P}}{t_{\mathrm{ge}}}\right)\right]^{-1} \\
C_{0 d} & =C_{0} g\left(\frac{X_{P}}{t_{\mathrm{ge}}}+\frac{\tilde{X}_{P}}{t_{\mathrm{ge}}}\right)
\end{aligned}
$$

respectively, where the electrostatic force is given as

$$
F_{R}=\sqrt{5} \frac{C_{0} V_{\mathrm{DC}}^{2}}{2 t_{\mathrm{ge}}} g^{\prime}\left(\frac{X_{P}}{t_{\mathrm{ge}}}+\frac{\tilde{X}_{P}}{t_{\mathrm{ge}}}\right) .
$$

\section{Validation of the Model}

The finite-element analysis (FEA) results of an $\mathrm{M}^{3}$ CMUT with $35-\mu \mathrm{m}$ radius are reported in [8]. The plate thicknesses and gap height, which are the layer dimensions in PolyMUMPs process, are given in [8, Table 1]. The material properties of polysilicon are also given right after Table 1 as $Y_{0}=158 \pm 10 \mathrm{GPa}, \sigma=0.22 \pm 0.01$, and $\rho=2328 \mathrm{~kg} / \mathrm{m}^{3}$. The analytical model presented in this paper is validated with the FEA results reported in [8]. The relevant data for model calculations are determined from [8] as $t_{\mathrm{ge}}=750 \pm 80 \mathrm{~nm}$, $t_{m}=1.5 \pm 0.1 \mu \mathrm{m}$, and $\tilde{t}_{m}=2 \pm 0.15 \mu \mathrm{m}$.

Using the median material and geometry data, the compliances are found as $C_{R m}=7.83 \times 10^{-5} \mathrm{~m} / \mathrm{Nt}$ and $\tilde{C}_{R m}=$ $3.30 \times 10^{-5} \mathrm{~m} / \mathrm{Nt}$, and $V_{r}$ as $172.2 \mathrm{~V}$. It is pointed out that the release holes were ignored in FEA and the plates are assumed initially flat, i.e., $F_{b} / F_{g}=0$. With $135 \mathrm{~V}$ bias, $V_{\mathrm{dc}} / V_{r}=0.784$, and CBC, (13) yields $X_{P}+\tilde{X}_{P}=0.1707 t_{\mathrm{ge}}$ for this bias level. The static transduction force, $F_{R}$, is found as $5.12 \times 10^{-4} \mathrm{Nt}$ under same conditions, using (17). The center deflections of the front and back plates are determined as 90 and $38 \mathrm{~nm}$, respectively, using (7) and (8), which matches to the FEA results reported in [8, Fig. 3]. The calculations are repeated for bias voltages changing between 10 and $150 \mathrm{~V}$, and the FEA results reported in [8, Fig. 4] are obtained from the model.

The masses of the plates (inductances in mechanical section) are found as $1.34 \times 10^{-11}$ and $1.79 \times 10^{-11} \mathrm{~kg}$ for $L_{R m}$ and $\tilde{L}_{R m}$, respectively. The mechanical resonance frequencies of 
the front and back plates are found in vacuum as 4.907 and $6.543 \mathrm{MHz}$, respectively, using the respective plate compliances and masses. When combined in series with $-C_{\mathrm{RS}}$, which is $-4.58 \times 10^{-4} \mathrm{~m} / \mathrm{Nt}$ at $135 \mathrm{~V}$ bias, the combined mechanical section on the right of electromechanical transformer in Fig. 3 has a resonance at $4.397 \mathrm{MHz}$.

The two mechanical resonators are coupled through the transduction force generated in the gap, since the displacement in each branch adds up to modify the force. This phenomenon dominates the transient response, unlike the static analysis, when there is no mechanical loss in the system. The Q factors of mechanical branches are more than 200 at $4.397 \mathrm{MHz}$ if the only loss is radiation resistance. Loss mechanisms like radiation resistance at each acoustic port or frictional resistance within the CMUT cell decrease the displacement amplitude in each plate and mitigate the effects arising from coupling at steady state. The time required to reach steady state is related to the time constants of the system and can be very large for low radiation resistance levels.

Transient response of lossless airborne CMUT cannot be usefully evaluated using FEA because of long transient responses and convergence problems while it is a straightforward circuit simulation in lumped element models. Large-signal equivalent circuit is implemented in $\mathrm{ADS}^{3}$ circuit simulator, and the transient response of the same $\mathrm{M}^{3}$ CMUT cell is simulated at $4 \mathrm{MHz}$. The response reaches to steady state in about $100 \mu \mathrm{s}$, whereas only $1.5 \mu \mathrm{s}$ of transient response is given in [8]. It is observed that the nonlinear effects become dominant at drive voltage levels exceeding $1 \mathrm{~V}$ peak, where 40-nm swing is already generated in the upper plate, and collapse occurs at about $7.5 \mathrm{~V}$. The exact conditions and precautions, like introduced loss for convergence, used in FEA analysis are not described in [8]. When a significant amount of extra loss in addition to radiation resistance is included in the equivalent circuit, transient response with amplitude levels similar to the one depicted in [8, Fig. 5] is observed. The first few cycles in the initial $1.5 \mu$ s of the transient response that are depicted in [8, Fig. 5] can only be qualitatively assessed to be in agreement.

The plates produced in PolyMUMPs process have release holes. The release holes are ignored in the FEA in [8] and in our analysis. The release holes have two effects on the plate: 1) the compliances of the plates increase, which is effective in static and dynamic performance and 2) they cause a very significant loss due to air friction, compared with radiation resistance during dynamic operation. The experimental measurements have lower collapse voltage and lower resonance frequencies compared with FEA prediction due to the effect given above in 1) and a wider bandwidth and reasonably short transient response in dynamic analysis due to extra loss effect described in 2).

\section{Coupling Coefficient}

The coupling coefficient in bilateral CMUT cell can readily be obtained using the approach given in [12]. When

\footnotetext{
${ }^{3}$ Advanced Design System, Agilent Technologies, Inc., Santa Clara, CA, USA.
}

the coupling coefficient definition of the square root of the of ratio of converted energy to the stored energy given in [12, eq. (4)] is adopted, dynamic coupling coefficient becomes

$$
k=\frac{1}{\sqrt{1+\frac{C_{0 d}}{n_{R}^{2}} \frac{C_{R S}-\left(C_{R m}+\tilde{C}_{R m}\right)}{C_{R S}\left(C_{R m}+\tilde{C}_{R m}\right)}}}
$$

in the absence of any parasitic capacitance, using the smallsignal equivalent circuit. If there is parasitic capacitance to be taken into account, $C_{0 d}$ must be augmented accordingly as in $[12$, eq. (8)]. The coupling coefficient increases to unity at the collapse threshold.

\section{Substrate AS BACKING IMPEDANCE}

The plate on one face is designed for radiation in a singlesided CMUT cell, whereas the other is the substrate, which is a very thick layer of a hard material. The impedance presented to the equivalent circuit by this face can be determined by increasing the thickness of the corresponding plate. If the thickness of the plate at the back (bottom) face is gradually increased, the compliance, $\tilde{C}_{R m}$, and the displacement, $\tilde{x}_{R}$, in that branch decrease. The compliance, $\tilde{C}_{R m}$, becomes very small and dominates the branch impedance.

If $\tilde{x}_{R}$ becomes insignificant compared with $x_{R}$, we can consider that a rigid backing terminates the gap and remove the branch from the circuit. However, $\tilde{x}_{R}$ can never be zero with real materials. The limiting case is an infinitely thick substrate.

Assume we use a block of some solid material and a CMUT is implemented on this block, which is the common assumption in CMUT models, analytic or numerical. The radiating plate is on one side and a substrate on the other.

A CMUT cell radiating into air or water has significantly smaller relative aperture on the substrate side. For example, consider an airborne CMUT cell with a reasonably large aperture, $k a=3$, build on borosilicate glass [13]. The velocities of sound in air and in borosilicate glass (longitudinal) are 340 and $5590 \mathrm{~m} / \mathrm{s}$, respectively. As far as the aperture on borosilicate glass is considered, $k a$ becomes 0.18 . This is like a point source radiating spherical waves into a solid half-space.

The acoustic impedance of spherical waves in isotropic solids is studied in [14]. Blake [14] derives the impedance as

$$
Z_{R b}=S \rho_{b} c_{b}\left\{R_{1 b}(k a)+j X_{1 b}(k a)\right\}
$$

where $\rho_{b}$ and $c_{b}$ are the density and the velocity of sound in the solid, $S$ is the area of the radiating surface, and $a$ is the radius. The normalized radiation resistance is given by

$$
R_{1 b}(k a)=\frac{(k a)^{2}}{1+(k a)^{2}}
$$

similar to $R_{1}(k a)$ of fluid medium. The reactance is significantly different and is derived as

$$
X_{1 b}(k a)=-\frac{1+(1-K)(k a)^{2}}{K(k a)\left[1+(k a)^{2}\right]}
$$




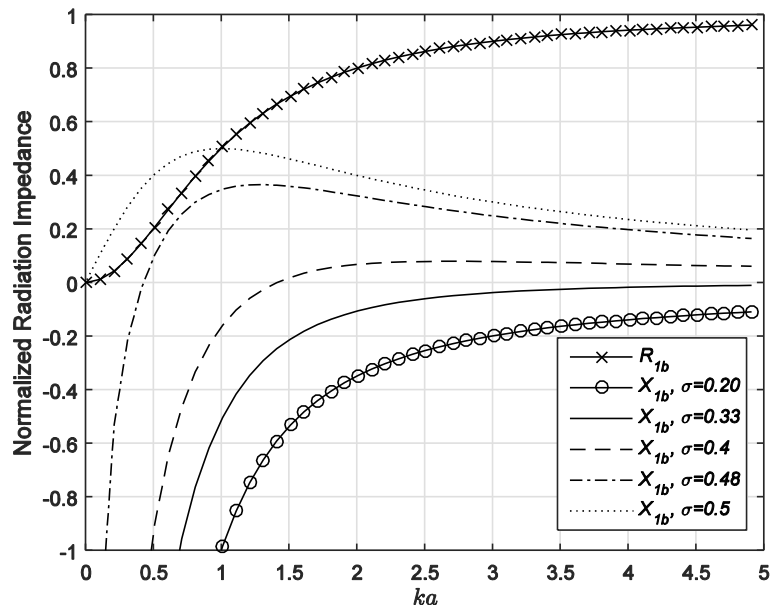

Fig. 4. Normalized radiation resistance and reactance of spherical waves in solids. The reactance is plotted for different values of the Poisson's ratio.

where

$$
K=\frac{1}{2} \frac{1-\sigma}{1-2 \sigma}
$$

and $\sigma$ is the Poisson's ratio in the solid. The radiation resistance and reactance are plotted with respect to $k a$ in Fig. 4, for different values of Poisson's ratio.

When $\sigma$ is in vicinity of 0.5 , which means that the medium is liquid, radiation reactance variation with respect to $k a$ is similar to that of water or air. Poisson's ratio is close to 0.5 also in soft polymers. Reactance is compliant only for very small values of $k a$ and becomes similar to fluid for larger $k a$ ( $\sigma=0.48$ depicted in Fig. 4). Reactance assumes negative values for small $k a$ values and positive for larger, when $1 / 3<\sigma<0.5$. Note that the radiation impedance has a series resonance at a particular value of $k a$ in this region.

The reactance is always compliant when $\sigma<1 / 3$. Poisson's ratio for borosilicate glass is 0.2 , and therefore yields a compliant reactance for all values of $k a$.

The reactance, $X_{1 b}(k a)$, can be decomposed as

$$
X_{1 b}(k a)=\frac{k a}{1+(k a)^{2}}-\frac{1}{K} \frac{1}{k a}=X_{1}(k a)-\frac{1}{K \frac{a}{c}} \frac{1}{\omega}
$$

where

$$
X_{1}(k a)=\frac{k a}{1+(k a)^{2}}
$$

which is the same as that of fluid. The backing impedance can now be written as

$$
Z_{R b}=S \rho c R_{1}(k a)+j S \rho c X_{1}(k a)+\frac{1}{j \omega} \frac{S \rho c}{K^{\frac{a}{c}}}
$$

where $Z_{R b}$ is the series combination of a component similar to the radiation impedance similar to that of a fluid (the same morphology) and a compliance, where

$$
C_{R b}=\frac{K a}{S \rho c^{2}}=\frac{\left(1+\sigma_{b}\right)}{2 \pi a Y_{0 b}}
$$

is the series compliance, where $Y_{0 b}$ and $\sigma_{b}$ are the Young's modulus and Poisson's ratio of the substrate material, respectively. The equivalent circuit including the backing impedance is shown in Fig. 5.
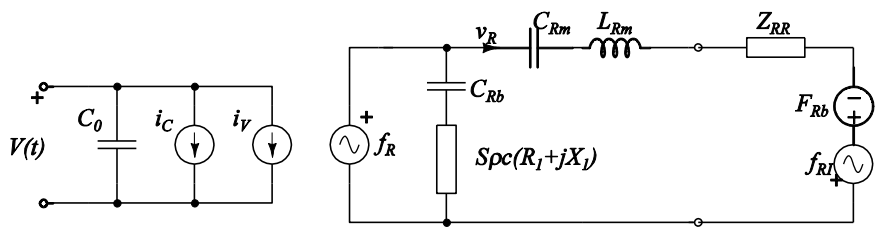

Fig. 5. Equivalent circuit model with infinite solid backing.

For an airborne CMUT cell, the reactance of $Z_{R b}$ is very large compared with the impedance of the front (air) segment. Hence, the rigid substrate assumption is justified.

The compliance of a clamped plate in CMUT [2] is very large when compared with $C_{R b}$ in the backing impedance. The ratio of two compliances can be written as

$$
\frac{C_{R m}}{C_{R b}}=\frac{9\left(1-\sigma^{2}\right)}{40\left(1+\sigma_{b}\right)} \frac{Y_{0 b}}{Y_{0}} \frac{a^{3}}{t_{m}^{3}} \text { for } \frac{a}{t_{m}}>8
$$

where $Y_{0}$ and $\sigma$ are the Young's modulus and Poisson's ratio of the plate, respectively. The plate compliance can be more than 100 times larger even for very thick plates with $a / t_{m}=8$, for plates and substrate made of the same material. The rigid backing assumption is well justified for an airborne CMUT with thick substrate.

\section{Symmetric Bilateral CMUT Cell}

\section{A. Unbaffled CMUT}

When the radiation plate on either side is the same, the bilateral CMUT becomes symmetrical. The symmetric bilateral CMUT radiates in exactly the same way at either side, except the direction of radiation is opposite. Therefore, a full symmetry is established in the acoustic fields of two half-spaces on either side of the CMUT's midplane.

As part of this symmetry, the normal component of particle velocity must vanish on the midplane, which is the rigid boundary condition. Even if the CMUTs are not in a baffle, midplane behaves as a rigid baffle. The practical design constraints ensure that the sum of the plate thickness and the insulation layer thickness, and the gap height is very small compared with the wavelength in the immersion medium at the operation frequency. Acoustically both radiating faces of the bilateral cell can be considered to be on the midplane because of this dimensional property

$$
2 t_{m}+2 t_{i}+t_{g} \ll \lambda
$$

Hence, both faces can be considered in rigid baffle, even when there is no baffle at all. The same arguments are also valid for symmetric bilateral CMUT arrays if all cells are symmetrically driven.

The model presented in this paper is also accurate for such symmetric bilateral CMUT cells and arrays without any rigid baffle.

The pressure directivity pattern $D(\theta)$ of a CMUT cell in rigid baffle is given as [15], [16]

$$
D(\theta, k a)=48 \frac{J_{3}(k a \sin (\theta))}{(k a \sin (\theta))^{3}}
$$




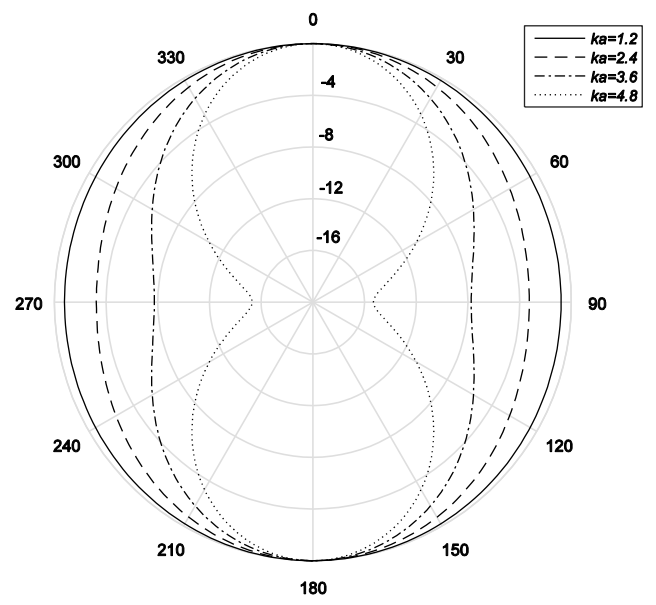

Fig. 6. Directivity pattern of symmetric bilateral CMUT for $k a=1.2,2.4$, 3.6, and 4.8. Radial scale is logarithmic, in decibels.

where $\theta$ is the direction of propagation relative to the normal to the plane of the cell. The same directivity pattern can be used for symmetric bilateral cells in two half-spaces. The directivity pattern is shown in Fig. 6 for various values of $k a$. The directivity pattern is quite omnidirectional even for $k a$ as large as 2.4. The on-axis radiation is only $3 \mathrm{~dB}$ higher compared with the radiation in the midplane. Waterborne CMUTs can be designed to be resonant at very low $k a$ values, unlike, for example, spherical PZT transducers.

\section{B. Diffraction Constant of CMUT in Rigid Baffle}

Transducers with uniform surface velocity are extensively studied [10], [17]-[21]. It is known that the diffraction constant, radiation resistance, and the directivity factor of such transducers are interrelated [10]. In [18], it is shown that the same relation is also satisfied by transducers with nonuniform but fixed surface velocity distribution. However, the lumped surface velocity, referred to as "reference" velocity, must be carefully defined in this case. Following the approach in [10] and [18], this relation for circular CMUT cell in rigid baffle is derived as:

$$
D_{a}^{2}(k a)=\frac{4 \pi R_{R A}(k a) D_{f}}{\rho c k^{2}\left(\pi a^{2}\right)^{2}}=\frac{4(1.8) R_{1}(k a) D_{f}}{(k a)^{2}}
$$

where $D_{a}$ is the diffraction constant, $R_{R A}$ and $R_{1}$ are the radiation resistance defined for average velocity given in [16], [22], and [23] as

$$
R_{R A}(k a)=1.8\left(\pi a^{2} \rho c\right) R_{1}(k a)
$$

and

$$
R_{1}(k a)=1-\frac{200}{(k a)^{9}} F_{1}(2 k a)
$$

and $D_{f}$ is the directivity factor of the CMUT cell, respectively. $D_{f}$ is calculated using the pressure directivity pattern, $D(\theta)$, given in (29). The directivity factor can be written as

$$
D_{f}=\left.D_{f}(\theta, k a)\right|_{\theta=0}=\left.\frac{D^{2}(\theta, k a)}{\frac{2 \pi}{4 \pi} \int_{0}^{\pi / 2} D^{2}(\gamma, k a) \sin \gamma d \gamma}\right|_{\theta=0} .
$$

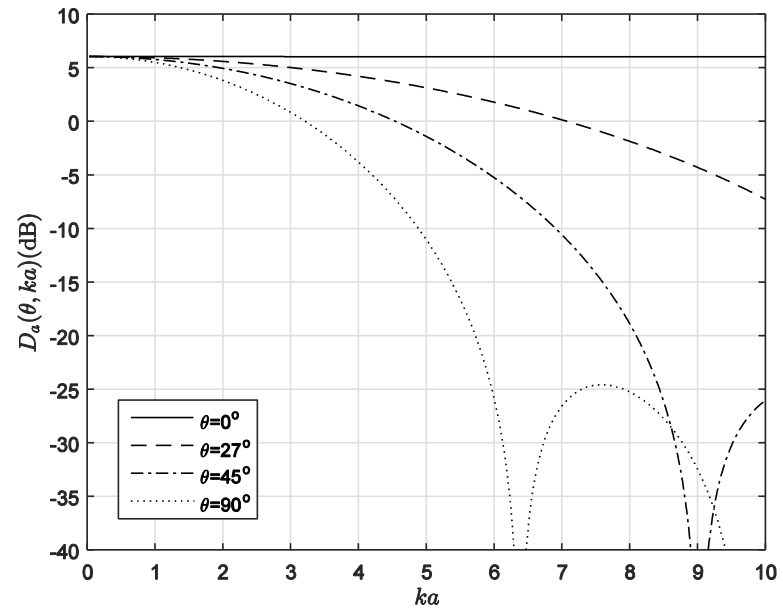

(a)

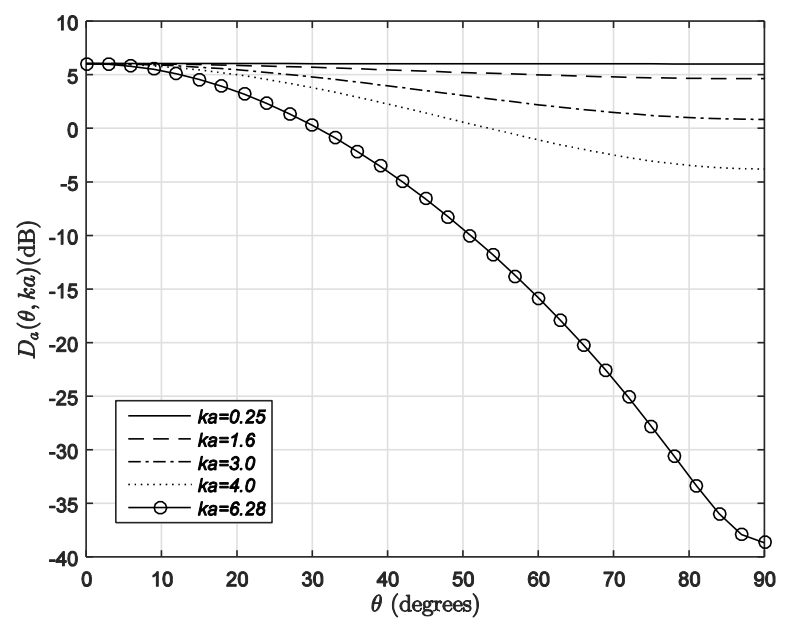

(b)

Fig. 7. Directional plane wave diffraction constant for a CMUT cell in rigid baffle. (a) Diffraction constant versus $k a$ for incidence angle $\theta=0^{\circ}$ (normal), $27^{\circ}, 45^{\circ}$, and $90^{\circ}$. (b) Diffraction constant versus incidence angle for $k a=0.25,1.6,3,4$, and 6.28 .

$D_{a}(k a)$ turns out to be equal to two for all values of $k a$, as expected. The directional plane wave diffraction constant can be calculated following the definition and the approach given in [19] as:

$$
D_{a}^{2}(\theta, k a)=\frac{4(1.8) R_{1}(k a) D_{f}(\theta, k a)}{(k a)^{2}} .
$$

The diffraction constant is depicted in Fig. 7 for different values of $k a$ and incidence angle. The diffraction coefficient is close to two for small $k a$ for all incidence angles. The minimum value of diffraction constant remains near two for moderate $k a$ values in CMUT cell. For example, $D_{a}>1.75$ for $k a=\pi / 2$.

This is the transmission diffraction constant and is numerically equal to the receiving diffraction constant for cells in rigid baffle. The force applied on the CMUT surface due to incident pressure $p_{i}$ is given as

$$
f_{I}=D_{a}(\theta, k a) \pi a^{2} p_{i} .
$$

The transmitting diffraction coefficient is also the same for symmetric bilateral cells in two half-spaces. 


\section{Receiving Unbaffled Symmetrical Bilateral CMUT}

When used as a hydrophone or a microphone, the lack of rigid baffle must be taken into account. The pressure directivity pattern and the radiation resistance of the unbaffled symmetric bilateral CMUT are required in order to calculate the relevant receiving diffraction coefficient. These two parameters are not readily available analytically, but can be numerically evaluated. However, an approximate measure can be defined by the help of prior work on thin disk hydrophones. The diffraction constant of thin rigid disk had been studied as early as 1932 for small $k a$ [24]. Spence [25] extended this work for analytic calculation of diffraction constant for all $k a$ values. Woollett [18] calculated the diffraction constant of flexural disk type pressure gradient transducers including the effect of circular inertial frame that holds the disk. Only the scattered field contributes to transduction in such transducers, and the diffraction constant is about $0.5 \mathrm{ka}$ for small $\mathrm{ka}$ if the frame diameter is close to the disk diameter. Diffraction coefficient increases for the same $k a$ as the frame diameter becomes larger.

On the other hand, it is known that $D_{a}$ of an unbaffled piston transducer mounted to the end of a long rigid rod is unity for $k a \ll 1$ [20]. As $k a$ increases, $D_{a}$ also monotonically increases to its upper limit of two for very large $k a . D_{a}$ is a measure of how much of incident pressure a receiving transducer can make use of. For example, the diffraction constant of spherical transducers is also unity for very small $k a$, but falls off rapidly as $k a$ increases [20].

The velocity profile on the CMUT's radiation plate is clamped disk profile and results in higher $k a$ values for equivalent radiation resistance and pressure directivity levels, compared with uniform and simply supported profiles, examined in the literature for diffraction coefficient. An accurate approximate receiving diffraction constant, $D_{a_{r}}(\theta, k a)$, can be conjectured as

$$
D_{a_{r}}(\theta, k a) \approx \begin{cases}D_{a}(\theta, k a), & \text { for } k a>3 \\ 1, & \text { for } k a<1.6\end{cases}
$$

A bilinear interpolation can be employed between 1 and $D_{a}(\theta, k a)$, the transmitting diffraction pattern, for $1.6<k a<3$ and for different values of $\theta$. It is important to note that diffraction constant can be taken as unity for $k a<1.6$ in all directions, for unbaffled symmetric bilateral CMUT cell. The forces applied on the surfaces become

$$
f_{I} \approx \tilde{f}_{I} \approx \pi a^{2} p_{i}
$$

in this useful $k a$ range.

\section{Array of Bilateral CMUT Cells}

\section{A. Small-Signal Equivalent Circuit for an Array of Bilateral CMUT Cells}

We consider $N$ bilateral cells connected to form an array of $m$ elements. Array elements are excited with voltage sources, $V_{\text {in } 1}, V_{\mathrm{in} 2}, \ldots, V_{\mathrm{inN}}$, each with a source resistance of $R_{T}$. The series mechanical loss resistances, $R_{A}$ and $\tilde{R}_{A}$, are included into each mechanical branch to model the frictional or other loss mechanisms, which limit the amplitude

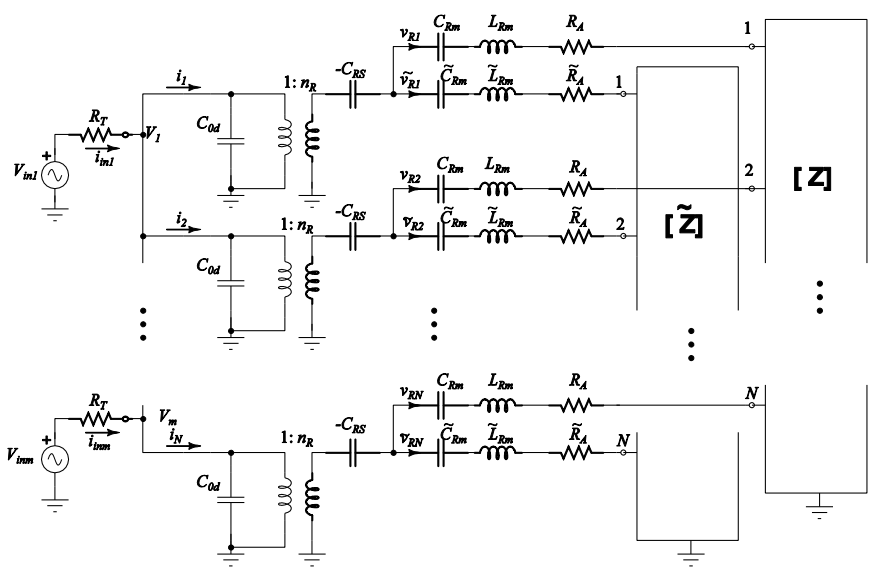

Fig. 8. Small-signal linear model of an array of bilateral CMUT cells.

of spurious Rayleigh-Bloch (RB) wave resonance vibrations [26]. As shown in Fig. 8, the element currents are $i_{\text {in } 1}, i_{\text {in } 2}, \ldots, i_{\text {inm }}$, while the individual cell currents are $i_{1}, i_{2}, \ldots, i_{N}$. The front and back plate peak velocities are shown with $v_{R 1}, v_{R 2}, \ldots, v_{R N}$ and $\tilde{v}_{R 1}, \tilde{v}_{R 2}, \ldots, \tilde{v}_{R N}$, respectively. The radiation impedance matrices for front $Z$ and back surfaces $\tilde{Z}$ are uncoupled, since the front and back immersion media are not acoustically coupled in an array of bilateral cells. With a sinusoidal excitation of the elements, $2 N$ unknown plate velocities at a given frequency can be solved by the inversion of a $2 N \times 2 N$ matrix, similar to what is done in [26].

\section{B. Effect of Back Plate Thickness on Reception Performance}

A single-element array of $7 \times 7$ cells, each with a radius $a=22 \mu \mathrm{m}$ and a front plate thickness $t_{m}$ of $1.5 \mu \mathrm{m}\left(a / t_{m}=\right.$ 14.7), is simulated in water for three different back plate thicknesses, 2,3 , and $4 \mu \mathrm{m}\left(a / \tilde{t}_{m}=11,7.3,5.5\right)$. The smallsignal equivalent circuit is used for the simulation of receiving cells, since the small-signal conditions are satisfied both on the electrical and the mechanical variables during reception. The plate constants are $Y_{0}=149 \mathrm{GPa}, \rho=2370$, and $\sigma=0.17$. The effective gap height is $95 \mathrm{~nm}$ and the cells are biased at $80 \%$ of their respective collapse voltages, where the standard atmospheric pressure is assumed for ambient pressure.

The short-circuit received current sensitivity in $\mathrm{A} / \mathrm{Pa}$ is plotted in Fig. 9 for three back plate thicknesses. The series loss resistance in both branches is assumed to be $R_{A}=$ $0.03 \pi a^{2} Z_{w}$ [26], where $Z_{w}$ is the specific acoustic impedance of water $\left(1.5 \times 10^{6} \mathrm{Nt} / \mathrm{m}^{3} / \mathrm{s}\right)$. This amount of series mechanical loss causes a decrease of $1 \mathrm{~dB}$ in the output current in all three devices. Even with such a loss, the RB wave resonance effects are still clearly observable at frequencies below $5 \mathrm{MHz}$. When the arrays are operated in pulsed mode, the effect of these resonances is extended low amplitude ringing in time domain [26], the duration of which is determined by the quality of the resonance.

The reception sensitivity slightly improves (less than $2 \mathrm{~dB}$ ) for thicker back plate due to larger bias voltage, since the cells with thicker back plate have larger collapse voltages. If the same bias voltage of $13 \mathrm{~V}, 0.8 V_{C}$ of the CMUT 


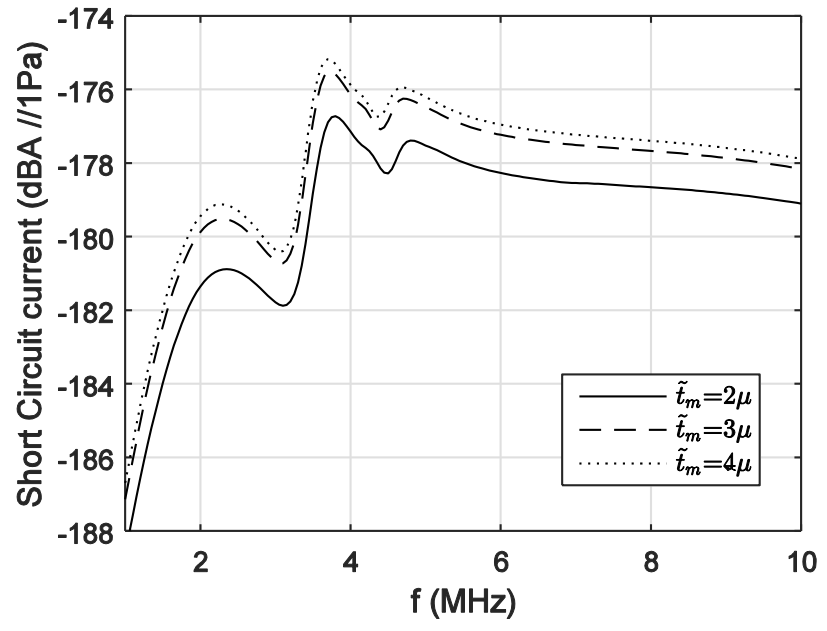

Fig. 9. Received short-circuit current response of a bilateral waterborne CMUT element of $7 \times 7$ cells with differing back plate thicknesses, when 1-Pa amplitude pressure wave is incident on the front surface. The cell dimensions are $a=22 \mu \mathrm{m}, t_{m}=1.5 \mu \mathrm{m}$ and $t_{\mathrm{ge}}=95 \mu \mathrm{nm}$. The spacing between the cells, $s$, is $3 \mu \mathrm{m}$, ambient pressure, $P_{0}$, is SAP, and $V_{\mathrm{dc}}=0.8 V_{C}$. The normalized series loss resistance $\tilde{R}_{A}$ is taken as 0.03 .

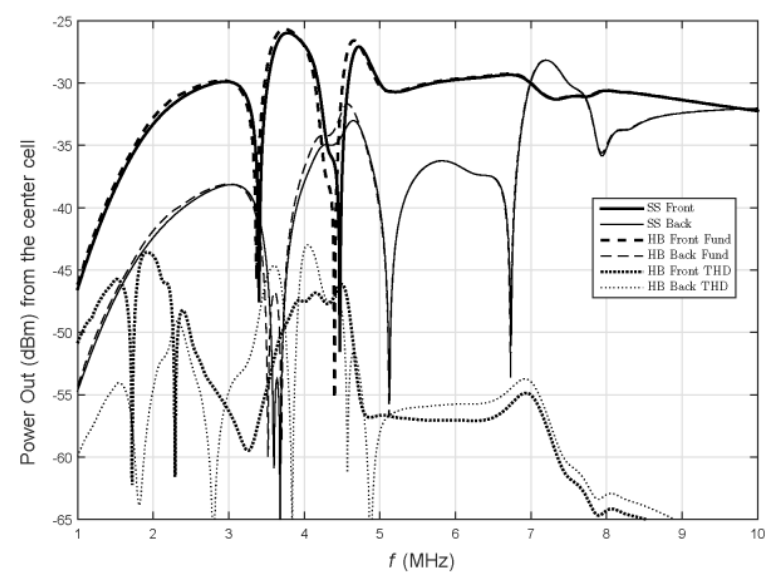

Fig. 10. Radiated power from the center cell of $7 \times 7$ element when the bias is $0.8 V_{C}$ and drive voltage is $1.8 \mathrm{~V}$ peak. The cell dimensions are $a=22 \mu \mathrm{m}$, $t_{m}=1.5 \mu \mathrm{m}, \tilde{t}_{m}=2 \mu \mathrm{m}$, and $t_{\mathrm{ge}}=95 \mathrm{~nm}$. The spacing between the cells $s$ is $3 \mu \mathrm{m}$, and ambient pressure $P_{0}$ is SAP. The normalized series loss resistance $\tilde{R}_{A}$ is taken as 0.03 .

with $2-\mu \mathrm{m}$ back plate, is used for all cells, the array with thinner back plate is most sensitive, $1.5 \mathrm{~dB}$ higher than the response of the thickest one. When the back plate is replaced by rigid substrate, reception performance is similar to that of $4-\mu \mathrm{m}$-thick back plate in the frequency band of interest.

\section{Effect of Back Plate Thickness on Radiation Performance}

The ac drive voltage and the mechanical displacement of the plate usually exceed the limits of small-signal conditions in transmitting CMUTs. The large-signal model is required for transmission simulations, especially if the amount of harmonic distortion is to be determined. The transmit response is also affected by RB wave resonances, which causes further ambiguity on the response when combined by the nonlinear nature of the CMUT.

In Fig. 10, the power radiated by the center cell of the $7 \times 7$ array element with $2-\mu \mathrm{m}$-thick back plate is depicted when the bias voltage is $0.8 V_{C}$ and ac drive voltage is a $1.8 \mathrm{~V}$

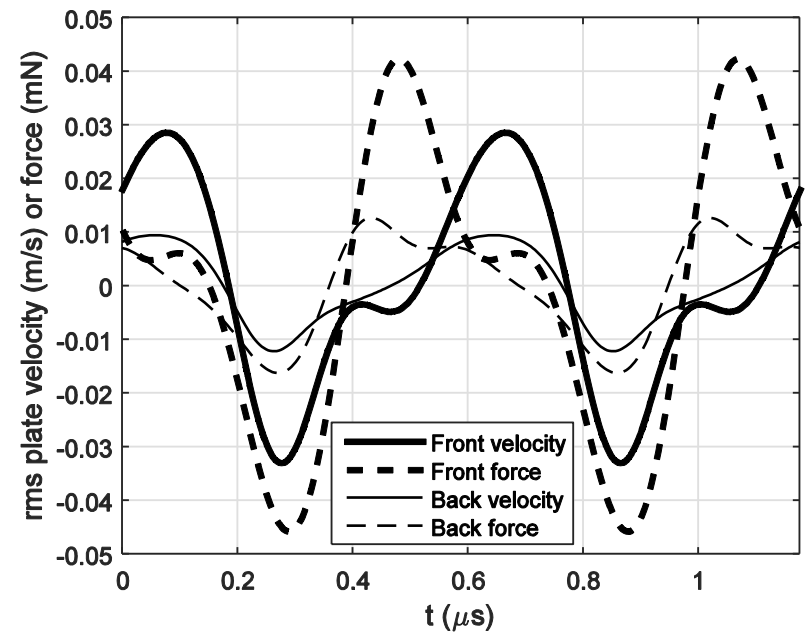

Fig. 11. Time waveform of the force and the velocity at front and back plates of the center cell at $1.7 \mathrm{MHz}$.

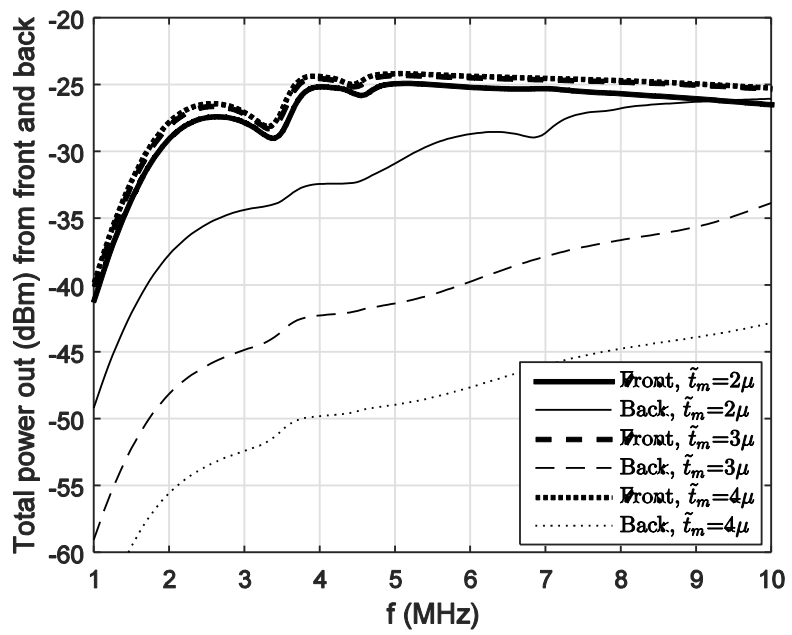

Fig. 12. Radiated power to the front and back half-space by a bilateral waterborne CMUT element of $7 \times 7$ cells with differing back plate thicknesses.

amplitude sine wave. The CMUT cell's response is highly nonlinear under this drive condition. The fundamental component power for front and back plates and the total harmonic distortion (THD) obtained by harmonic balance analysis using ADS circuit simulation software are plotted together with the response using small-signal model. The power in the fundamental component follows the linear response very closely at all frequencies both for front and back plates, although there is very significant amount of THD, particularly at the back plate. At $3.6 \mathrm{MHz}$, the THD is more than the fundamental power. THD is dominated by the second harmonic power, and a highly resonant behavior in THD is clearly observed at the frequency range, which is half of that of $\mathrm{RB}$ wave resonances occur in linear operation.

The velocity of the front and back plates of the center cell and force on the plates at $1.7 \mathrm{MHz}$ are shown in Fig. 11 . The nonlinear distortion in all signals is significant.

Radiated power to the front and back half-space is plotted in Fig. 12 using the small-signal model. The cells are biased 
again at $80 \%$ of their respective collapse voltages and driven by a sinusoidal signal of $500-\mathrm{mV}$ amplitude from a $50-\Omega$ source (available power is $-2 \mathrm{dBm}$ ). The RB wave resonances are visible in the front radiated power at frequencies below $5 \mathrm{MHz}$ albeit at a much lower amplitude. Similarly, RB wave resonances exist at the back surface (e.g., at $6.9 \mathrm{MHz}$ for $\tilde{t}_{m}=2 \mu \mathrm{m}$ ). Note that these resonances weakly couple to the opposing surface. Since the total radiated power is contributed by all CMUT cells behaving differently, the strong RB wave resonances of the single cell seen in Fig. 10 tend to be washed out. There is about $25-\mathrm{dB}$ difference at $5 \mathrm{MHz}$ between the front and back radiated power in the CMUT with the thickest back plate. When the back plate is replaced by a rigid substrate, the front radiation is similar to that of CMUTs with the thickest back plate, but radiation to the back hemisphere vanishes.

\section{CONCLUSION}

In this paper, we present the bilateral CMUT cell. A threeport equivalent circuit model is derived, and the effect of the back plate thickness is studied using this model. It is observed that the emission from the back plate is $25 \mathrm{~dB}$ lower in the passband for waterborne operation, when the back plate is 2.7 times thicker compared with the radiating plate and when the CMUT is biased at $80 \%$ of the collapse voltage.

Very high receiving sensitivity of CMUTs makes the unbaffled symmetric bilateral CMUT, which is also presented in this paper, a potential contender for omnidirectional hydrophone applications. Bilateral CMUT offers high sensitivity with a small dimension compared with wavelength. CMUT cells can be designed for wide-bandwidth operation at resonance at very low $k a$ values. This option makes CMUT hydrophones suitable for high-sensitivity hydrophone operation both at resonance and off resonance. Using symmetric bilateral CMUT in collapsed mode will provide a tradeoff between the sensitivity and ambient static pressure tolerance.

It is shown in this paper that the energy lost to the substrate can be ignored in airborne CMUTs with thick substrate, since the backing is essentially rigid. However, there may be multiple reflections of this very low energy due finite dimensions of the substrate, which may yield ringing. This low amplitude ringing interferes with the received signal in pulse-echo applications and limits the dynamic range. Using some absorbing material in contact with the substrate is instrumental to avoid the multiple reflections in the substrate.

The linear model is sufficient to characterize the fundamental component of the response, even at high excitation levels. Nonlinear model with harmonic balance simulation is needed to determine the level of the harmonic distortion components. RB wave resonances cause significant effects in the individual cell responses even in the presence of mechanical loss in the plates. However, their effect is reduced and averaged out when the outputs of many cells are summed as in the case of parallel connected cells forming an element.

\section{REFERENCES}

[1] M. I. Haller and B. T. Khuri-Yakub, "A surface micromachined electrostatic ultrasonic air transducer," IEEE Trans. Ultrason., Ferroelect., Freq. Control, vol. 43, no. 1, pp. 1-6, Jan. 1996.

[2] H. Koymen et al., "An improved lumped element nonlinear circuit model for a circular CMUT cell," IEEE Trans. Ultrason., Ferroelect., Freq. Control, vol. 59, no. 8, pp. 1791-1799, Aug. 2012.

[3] K. R. Chapagain and A. Ronnekleiv, "Grooved backing structure for CMUTs," IEEE Trans. Ultrason., Ferroelect., Freq. Control, vol. 60, no. 11, pp. 2440-2452, Nov. 2013.

[4] A. S. Savoia, M. L. Mura, B. Mauti, N. Lamberti, and G. Caliano, "Reverberation reduction in capacitive micromachined ultrasonic transducers (CMUTs) by front-face reflectivity minimization," in Proc. ICU Int. Congr. Ultrason., Metz, France, 2015, pp. 941-944.

[5] S. Berg and A. Ronnekleiv, "Acoustic backing in 3-D integration of CMUT with front-end electronics," IEEE Trans. Ultrason., Ferroelect., Freq. Control, vol. 59, no. 7, pp. 1537-1549, Jul. 2012.

[6] T. A. Emadi and D. A. Buchanan, "Multiple moving membrane CMUT with enlarged membrane displacement and low pull-down voltage," IEEE Electron Device Lett., vol. 34, no. 12, pp. 1578-1580, Dec. 2013.

[7] T. A. Emadi and D. A. Buchanan, "Capacitive micromachined ultrasonic transducer with multiple deflectable membranes," WO Patent 2014134723 A1, Sep. 12, 2014.

[8] T. A. Emadi and D. A. Buchanan, "Design and fabrication of a novel MEMS capacitive transducer with multiple moving membrane, $\mathrm{M}^{3}$-CMUT," IEEE Trans. Electron Devices, vol. 61, no. 3, pp. 890-896, Mar. 2014.

[9] (Mar. 2016). MEMSCAP. [Online]. Available: http://www.memscap. com/products/mumps/polymumps

[10] R. J. Bobber, "Diffraction constants of transducers," J. Acoust. Soc Amer, vol. 37, no. 6, pp. 591-595, 1965.

[11] D. Elata and H. Bamberger, "On the dynamic pull-in of electrostatic actuators with multiple degrees of freedom and multiple voltage sources," J. Microelectromech. Syst., vol. 15, no. 1, pp. 131-140, Feb. 2006

[12] H. Köymen, A. Atalar, and H. K. Oğuz, "Designing circular CMUT cells using CMUT biasing chart," in Proc. IEEE Int. Ultrason. Symp., Dresden, Germany, Oct. 2012, pp. 975-978.

[13] A. Unlugedik, A. S. Tasdelen, A. Atalar, and H. Koymen, "Designing transmitting CMUT cells for airborne applications," IEEE Trans. Ultrason., Ferroelect., Freq. Control, vol. 61, no. 11, pp. 1899-1910, Nov. 2014.

[14] F. G. Blake, Jr., "Spherical wave propagation in solid media," J. Acoust. Soc. Amer, vol. 24, no. 2, pp. 211-215, 1952.

[15] H. K. Oguz, A. Atalar, and H. Köymen, "Equivalent circuit-based analysis of CMUT cell dynamics in arrays," IEEE Trans. Ultrason., Ferroelect., Freq. Control, vol. 60, no. 5, pp. 1016-1024, May 2013.

[16] D. T. Porter, "Self- and mutual-radiation impedance and beam patterns for flexural disks in a rigid plane," J. Acoust. Soc. Amer., vol. 36, no. 6, pp. 1154-1161, 1964.

[17] C. C. Sims, "Standard calibration hydrophone," J. Acoust. Soc. Amer. vol. 31, no. 12, pp. 1676-1680, 1959.

[18] R. S. Woollett, "Diffraction constants for pressure gradient transducers," J. Acoust. Soc. Amer, vol. 72, no. 4, pp. 1105-1113, 1982.

[19] C. H. Sherman and J. L. Butler, Transducers and Arrays for Underwater Sound (The Underwater Acoustics Series). New York, NY, USA: Springer, 2007, pp. 490-493.

[20] T. A. Henriquez, "Diffraction constants of acoustic transducers," J. Acoust. Soc. Amer., vol. 36, no. 2, pp. 267-269, 1964.

[21] Z. Milošć, "Comments on 'Diffraction constants of acoustic transducers," J. Acoust. Soc. Amer., vol. 93, no. 2, p. 1202, 1993.

[22] H. K. Oguz, S. Olcum, M. N. Senlik, V. Tas, A. Atalar, and H. Koymen, "Nonlinear modeling of an immersed transmitting capacitive micromachined ultrasonic transducer for harmonic balance analysis," IEEE Trans. Ultrason., Ferroelect., Freq. Control, vol. 57, no. 2, pp. 438-447, Feb. 2010.

[23] M. Greenspan, "Piston radiator: Some extensions of the theory," J. Acoust. Soc. Amer., vol. 65, no. 3, pp. 608-621, 1979.

[24] H. Lamb, Hydrodynamics, 6th ed. Cambridge, U.K.: Cambridge Univ. Press, 1993, pp. 144-517.

[25] R. D. Spence, "The diffraction of sound by circular disks and apertures," J. Acoust. Soc. Amer., vol. 20, no. 4, pp. 360-380, 1948.

[26] A. Atalar, H. Köymen, and H. K. Oğuz, "Rayleigh-Bloch waves in CMUT arrays," IEEE Trans. Ultrason., Ferroelect., Freq. Control, vol. 61, no. 12, pp. 2139-2148, Dec. 2014. 


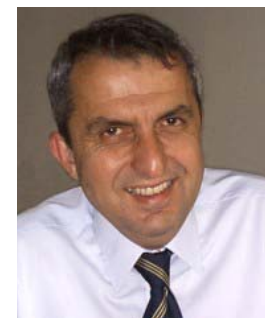

Hayrettin Köymen (S'74-M'76-SM'91) received the B.Sc. and M.Sc. degrees in electrical engineering from Middle East Technical University (METU), Ankara, Turkey, in 1973 and 1976, respectively, and the Ph.D. degree in electrical engineering from Birmingham University, Birmingham, U.K., in 1979.

From 1979 to 1990, he was with the Department of Marine Sciences, Mersin, METU, Turkey, and with the Department of Electrical Engineering, METU. In 1990, he joined the faculty of Bilkent University, Ankara, where he is currently a Professor with the Department of Electrical and Electronics Engineering. His research interests include underwater and airborne acoustic and ultrasonic transducer design, underwater acoustics, underwater and airborne acoustic systems, acoustic microscopy, ultrasonic NDT, biomedical instrumentation, mobile communications, and spectrum management.

Prof. Köymen is an IET Fellow.

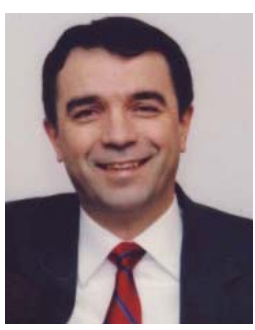

Abdullah Atalar (M'88-SM'90-F'07) received the B.S. degree in electrical engineering from Middle East Technical University (METU), Ankara, Turkey, in 1974, and the M.S. and Ph.D. degrees in electrical engineering from Stanford University, Stanford, CA, USA, in 1976 and 1978, respectively.

In 1979, he was with the Hewlett-Packard Laboratories, Palo Alto, CA, USA. From 1980 to 1986, he was an Assistant Professor with METU. In 1986, he was a Chairman with the Department of Electrical and Electronics Engineering, Bilkent University, Ankara, and participated in founding of the Department. In 1995, he was a Visiting Professor with Stanford University. From 1996 to 2010, he was the provost of Bilkent University, where he is currently the Rector and a Professor. His current research interests include micromachined devices and microwave electronics.

Dr. Atalar is a member of the Turkish Academy of Sciences. He was a recipient of the TUBITAK Science Award in 1994.

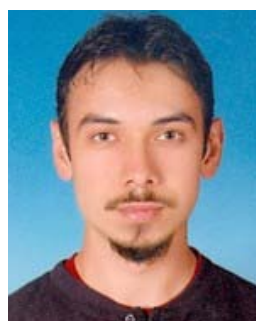

A. Sinan Taşdelen received the B.Sc. and M.Sc. degrees in electrical and electronics engineering from Bilkent University, Ankara, Turkey, in 2004 and 2007, respectively.

In 2008, he joined the Bilkent University Acoustics and Underwater Technologies Research Center, Bilkent University, where he is currently Head Research Engineer. His current research interests include passive radar, underwater acoustics, transducer design, and biomedical ultrasound. 